\title{
Zur Kongruenz des lappischen Adjektivattributs
}

Die für das Lappische charakteristische Halbkongruenz bei einem Beziehungswort im Inessiv steht das pronominale oder Zahlwortattribut im Essiv, bei einem Beziehungswort im Elativ im Partitiv, bei einem Beziehungswort im Mlativ im Lativ, in den anderen Kasus herrscht Vollkongruenz (Wiklund, JSFOu X 207ff) - hat PaAvo Ravila als eine archaische Erscheinung angesehen: sie sei eine Erbschaft aus der Zeit, als die allgemeinen Lokalkasus noch als Lokalkasus im Nomenparadigma verwendet wurden; auch die Kongruenz des Adjektivattributs der ostseefinnischen Sprachen sei auf eine ähnliche alte Kongruenz der Pronomina zurückzuführen (Sananjalka 2, 33ff). ERkKi Itkonen hat jedoch die Auffassung vertreten, dass die heutige Inkongruenz des lappischen Adjektivattributs sekundär sei und aus der Entwicklung der attributiven Form der Adjektive resultiere; die attributive Form kann nicht kongruieren. Itkonen zeigt, dass im Inarilappischen die Adjektivtypen, die keine attributive Form haben (nämlich die komparativischen, superlativischen, deminutivischen Adjektive), mit ihrem Beziehungswort kongruieren, wobei es sich ursprünglich um die Halbkongruenz gehandelt hat. Spuren dieser Kongruenz begegnen wenigstens in den komparativischen und deminutivischen Adjektiven des Skoltlappischen (CSIFU I 204ff). Diese Auffassung wird dadurch gestützt, dass auch im Süd- und Umelappischen Kongruenzfälle gefunden worden sind. Unter diesen Fällen sind komparativische, superlativische, einige auf Vokal endende und präsenspartizipförmige Adjektive vertreten (Knut Bergsland Gramm. S. 265, Stud.Sept. III 43, V 34; J. Qvigstad Stud.Sept. III 24; Bergsland ist jedoch der Ansicht, dass die Kongruenz sekundär sei, analog der der Pronomina entstanden). Einige Anzeichen scheinen darauf 
hinzudeuten, dass auch die heutzutage für das Inarilappische charakteristische Kongruenz der Deminutivadjektiva ( $ّ$ Adjektiva) früher viel verbreiteter gewesen ist.

Bergsland hat den norwegischlappischen Deminutivadjektiven schon früher Aufmerksamkeit geschenkt (Stud. Sept. II). Bergsland zeigt nämlich, dass man den früher als Charakteristikum des Ostlappischen angesehenen Schwund von *ń́ž vor geschlossener Silbe auch im Norwegischlappischen konstatieren kann; nur so können die Verhältnisse in den Formen $1 p \mathrm{~N}$ olgu-bal'dĕ $\sim \operatorname{lpN}$ Lenv. olguš-bal'dĕ 'draussen, von draussen', (j)ievt(a) akkĕd 'gestern Abend' (j)ievtaš 'gestriger' verstanden werden. Erstere Formen vertreten den Genitiv, letztere den Nominativ. Bergsland ist der Meinung, dass die Kongruenz auch in diesen Fällen sekundär sei und eine Analogie zu der Kongruenz der Pronomina vorliege (Stud.Sept. II 5ff). Ravila vertritt die Ansicht, dass das pronominale Attribut im Gen.-Akk. vor seinem Beziehungswort in einem Lokalkasus im Norwegischlappischen eine so junge Erscheinung sei, dass es nicht auf die Form des ersten Gliedes der oben angeführten Zusammensetzungen habe einwirken können. Nach Ravila lassen sich die Formen am natürlichsten so erklären, dass sie als Genitiv der Substantive, der substantivisch verwendeten Adjektive, gedeutet werden (FUF XXXIII 295ff).

Auch andere Spuren der Kongruenz der Deminutivadjektive ausserhalb des Ostlappischen erscheinen gerade in den von Bergsland und Ravila angeführten zusammengesetzten Partikeln. In ihnen tritt im zweiten Glied als Stamm balle- oder gaéče- auf, erstes Glied ist ein aus einem ein räumliches Verhältnis ausdrückenden defekten Stamm gebildetes Deminutivadjektiv. Den westlichen Typ vertritt olgǔ̌-, den östlichen olgu-, z.B.:

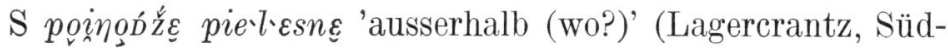
lapp.Wb. s.v.1591), лuwлačc pì (Lagercrantz, LV I 89); U nuor'tatja bielèsna 'an der Nordseite'

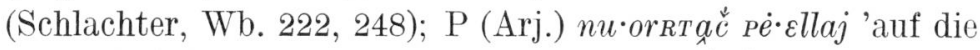
Nordseite' (Lagercrantz, LV I 45), nu•ornт a Nordseite' (ebd. I 37), ōrjačce-pé:cllaj 'auf die Südseite' (ebd. I 45), ōrjačc Péerllaj (ebd. I 45); L (Jokkm.) nuor'tasj pieleen 'auf 
der Nordseite' (Pirak, 166), åarjaasj pieleen 'auf der Südseite' (ebd. 26), (Nordf.) aluč ballai 'auf die Westseite' (Qvigstad, LES IV 512); N (Ibest.) olkusballai 'ausserhalb (wohin?)' (ebd. II 586), hier also $-s>-s$ wie in Jukkasjärvi und in Karesuando, Collinder, The Lappish Dialect of Jukkasjärvi S. 275, (Lenv.) olkušbal'de 'auf der Aussenseite' (Qvigstad, LES II 244), (Storfj.) olgošbcl'de (ebd. IV 58), abašbel'de 'ausserhalb (wo?, woher?)' (ebd. IV 210), (Kalfj.-Helg.) woarjaš bald 'auf der südlichen Seite' (Qvigstad, LTKH 28), olgušbal'de 'ausserhalb (wo?)' (ebd. 36).

Der entsprechende östliche Typ reicht bis Karesuando und in den Nordteil Gällivares:

L ålku-: NG *ilk $\omega$-, $\dot{o} l^{c} k \omega$ - (als erstes Glied in Zusammensetzungen) 'Aussen-'; ål 'ku-pǟèn 'auf der Aussenseite' (Grundström, Wb. 1495b); N (Kares.) sisku peälde 'auf der Innenseite, innerhalb' (Halász, SLNy VI 172), (Kautok.) davábællai 'auf die Nordseite' (A.Bær, 92), oriapcelte 'auf der Westseite' (Turi, Muitt. 78), sälkkapälte 'auf der Rückseite' (P.Turi, LT 102), (Alta) vuolabceld 'unterhalb (wo?)' (Qvigstad, LES II 570), (Talv.) vulubceld id. (ebd. II 390), (Inari) vūlo-pëällai 'unterhalb (wohin?)' (Ravila, RLVG 140), (Tana) siskuballai 'innerhalb (wohin?)' (Qvigstad, LES II 360), (Nes.) mânabcellai 'hinter (wohin?)' (ebd. I 476), gaddaballai 'auf die Landseite' (ebd. I 526), soggaballai 'auf die Wandseite' (ebd. I 412), uskabcellai 'auf die Türseite' (ebd. I 380), mìèða-pe:ällaj 'auf die Leeseite' (Lagercrantz, LV III 226), owdâballai 'auf die Vorderseite' (Qvigstad, LES 340), nuortâbcllai 'auf die Ostseite' (ebd. I 404), oustâbreld 'auf der Ostseite' (ebd. I 502), væstâbæeld 'auf der Westseite' (ebd. I 348), maddâbceld 'auf der Südseite' (ebd. I 332), siskabcld 'innerhalb (wo?)' (Qvigstad, LES I 284, 324, 326), olgubcel'de 'ausserhalb (wo?)' (ebd. I 484), (Kotaj.) mañä-вeälläi 'hinter (wohin?)' (Ravila, RLKN

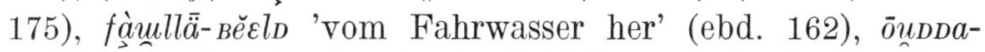

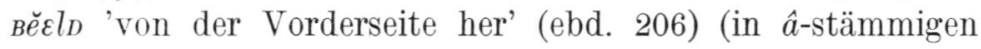
Wörtern begegnet man also $\hat{a}$ statt erwartungsgemässem $i$, was auf Analogie des Stammvokals des Grundwortes beruhen mag [Bergsland, Stud.Sept. II 7]; diese Formen könnten sich auch als Vertreter des in anderen Stammtypen im Norwegisch- und 
Inarilappischen nicht begegnenden Typs olgobcel'de erklären lassen, vgl. unten S. 36); I maajaa-peln 'hinter, nach (wo? wovon?)', taavaa-peln 'auf, von der Nordseite', maddáapeln 'auf, von der Südseite', ovdii-peln 'auf, von der Vorderseite', uskii-pel 'auf die 'Türseite', vuoluu-peln 'auf, von der Unterseite', ridduu-peln 'auf der Uferseite', vиoluи-keččn 'am unteren Ende', possuukečč in 'im innersten Teil' (Wb. [= Mskr. des Inarilappischen Wörterbuches]); R (Paatsj.) vŭsłă-bǔèln 'auf der Unterseite' (T. I. Itkonen, KKSK 368a), tàv ${ }^{a}$-вё̌̆̈lla, - $B^{i}$ èn 'auf die Nordseite, auf der Nordseite, von der Nordseite her' (ebd. 578a), m̨̌̌da-běăilla 'auf die Leeseite' (ebd. 255a), (Nuortij.) vupra-Bieln-skèüñe (ein Ohrzeichen des Rens) (ebd. 768a).

Als erstes Glied können in diesen zusammengesetzten Partikeln auch dieselben defekten unabgeleiteten Stämme auftreten sowie einige andere Substantive, z.B.:

Keine Beispiele aus dem Südlappischen; U (Malå) lùllègietjèsna 'am Ostende' (Schlachter, Wb. 182); P (Fauske) allegečin 'am Westende' (Qvigstad, LES IV 514); L (Jokkm.) lullee-pällài 'auf die Ostseite' (Pirak, 155), ålkì̀-pällài 'ausserhalb (wohin?)' (ebd. 57), pieivee-pällài 'auf die Südseite' (ebd. 4), mannee-kietjees 'von der Rückseite, Hinterseite' (ebd. 66); X (Ibest.) mannjeballai 'auf die hintere Seite' (Qvigstad, LES II 662), (Mand.) faw'lebcellai 'auf die Seite des Fahrwassers' (ebd. IV 80), (Storfj.) baddjebcellai 'oberhalb (wohin?)' (ebd. IV 256), davvebcellai 'ausserhalb (wohin?)' (ebd. IV 110), (Kåfj.) gad'deballai 'zwischen - und dem Land' (ebd. IV 40), (Kautok.) lullegeččai 'ans Südende' (A.Bær, 91) (Storfj.) baivibal'de 'auf der Südseite' (Qvigstad, LES IV 190), (Lyng.) sullo pé夭llaj 'an die Inselseite' (Lagercrantz, LV II 93), (Kautok.) smak'komuor-goadibcel'de 'zwischen Hackklotz und Zelt' (Qvigstad, LES II 714), (Nes.) su marâbeld 'zwischen ihm

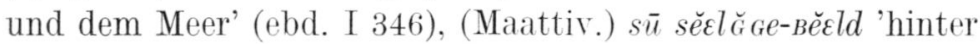
seinem Rücken' (Ravila, RLKN 56); I kezipeln 'auf, von der Südseite', seinipeln 'auf, von der Wandseite', selgipel 'auf die Rückseite' (Wb.); R (Paatsj.) pàeběăilla 'oberhalb (wohin?)' (T. I. Itkonen, KKS 62), mäebeln 'hinter (wo?)' (ebd. 130), (Suonik.) $\bar{o} u D^{n_{B}{ }^{i} e l n}$ 'voran, vor (wo?)' (ebd. 181), (Kild.) 


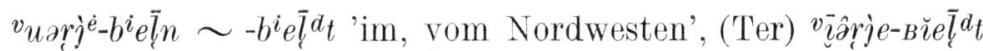
im, rom Norden' (T. I. Itkonen, KKSK 793a), sona sielçe *peält 'hinter ihm' (T. I. Itkonen, KKS 283).

Dass die Substantivbestimmung im Osten im Genitiv auftritt, scheint darauf hinzuweisen, dass man auch den ersten Bestandteil der Form olgubcel'de als substantivischen Genitiv auffassen kann. Dieser Wechsel zwischen Nominativ und Genitiv in den als Bestimmungslied vorkommenden Substantiven steht offensichtlich mit dem Wechsel der Deklinationstypen des Grundteils im Zusammenhang. Im Westen sind nämlich die Partikel ihrer Flexion nach ins Nomenparadigma übergegangen: S (Vefsn) p peăà̀n (Lagercrantz, Sprachl. 43), pì èlesne (Lagercrantz, LV I 89); U (Malå) biällàje (Schlachter, Wb. 265), bielèsna (ebd. 222); P (Arj.) p $\dot{e} \cdot \varepsilon l l a j$ (Lagercrantz, LV I 45), pè்̀ ·lièn (ebd. I 37); L (Jokkm.) pällài (Pirak, 155), pieleen (ebd. 166), pieleest (ebd. 169); N (Ibest.) bcelen (Qvigstad, LES II 454) (Kares.) pĕ̌̆lтe (Lagercrantz, LV II 196), (Lenv:) bal'de (Qvigstad, LES II 582), (Balsfj. bcel'de (ebd. II 6), (Sørfj.) bel'de (ebd. III 94), (Storfj.) bel'de (ebd.

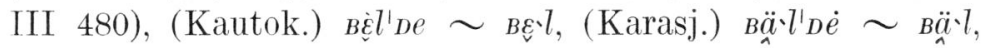

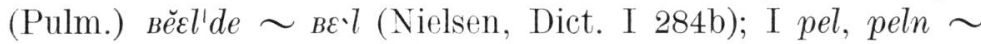
pen (Wb.); R (Paatsj.) běăilla, (Nuortij.) ве̌ă ̌̀łła, (Kild.) вёàtła, (Paatsj.) bǔę̄̆n, beln, (Nuortij.) вel̨n, abweichend von den anderen ostlappischen Vertretungen: (Kild.) $B^{i} e \bar{l} d_{t}$, (Tšernj.) *pielt, (Ter) (G.) *pie $i l t,(\mathrm{E}) * p e.(\ddot{a}) l t,(\mathrm{Akk}).(\mathrm{G}) * b. i e_{i} l t,(\mathrm{E}$. *piäld (T. I. Itkonen, KKSK 367b) // L (Jokkm.) kietjeen (Pirak, 55), kietjees (ebd. 1) 〜 N (Kautok.) Ǵähtšsen, (Karasj.)

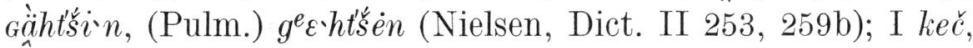
keččin (Wb.); R (Kild.) geačča (Genetz, Wb. 245), (Nuortij.) keštt'śèn (T. I. Itkonen, KKSK 124b). - Dieselbe Erscheinung wird auch in zwei anderen Partikeln sichtbar: S (Vefsn)

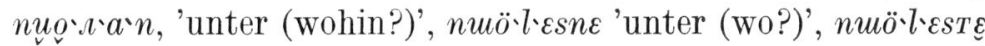
'von unter' (Lagercrantz, Südlapp.Wb. s.v. 1358); U (Malå) vuallàja (Schlachter, Wb. 153a), vüelèsna, vüelèsta (ebd. 156b); P (Arj.) vŭŏ. ìlaj, vù·òlièn (Lagercrantz, LW s. v. 1023), vûlēst, vûlest (Halász, SLNy VI 173); L vuollāi (Grundström, Wb. 1443b), vuolēn (ebd. 1441), vuolès (ebd. 1442a); N (Ofot.) vuolin (Qvigstad, LES IV 510), (Ibest.) vuolin (ebd. II 310) 
(Grat.) vŭọ̆ $\cdot \grave{l}_{T e}$, (Park.) vŭŏ $\cdot \grave{l}_{T e}$, (Könk.) vŭŏ $\cdot \grave{l}_{T e}$, (Enont.) vǔŏ $\grave{l}_{T e}$ (Lagercrantz, LW s.v. 1023), (Kautok.) vŭol'De

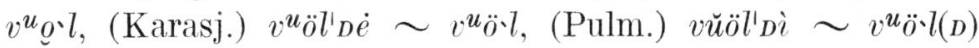
(Nielsen, Dict. III 803b); I vuäla vual, vyelni $\sim$ vyeln $(\mathrm{Wb}$.$) ;$

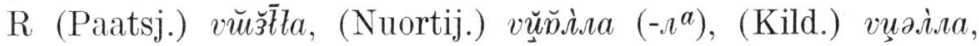
(Akk.) (G.) *volla, (R Fr.) valla, (Paatsj.) vŭă $\bar{\imath}(e)$, (Nuortij.)

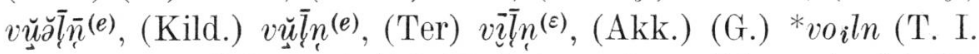
Itkonen, KKSK 785a) // S (Vefsn) tuok ka'n 'hinter (wohin?)', Dü̈ $(k s, n \varepsilon$ 'hinter (wo?)', tü̈kst 'hinter (hervor)' (Lagercrantz, Südlapp. Wb. s.v. 2669); U (Malå) Duahkàing (Schlachter, Wb. 36b), tuohkai (Budenz, NyK XII 166), Dự̆gèsna, Dü. Ğèsta (Schlachter, Wb. 36b); P (Arj.) тùò̀kièn (Lagercrantz, LV I 56), (Fauske) doahkai (Qvigstad, LES IV 518), duogin (ebd. IV 516); L tuohkāi (Grundström, Wb. 1256b), tuokēn (ebd. 1257a), tuokēs (ebd. 1257b); N (Park.) тù·òkien ${ }^{N}$ (Lagercrantz, LW s.v. 949), (Balsfj.) duogèn (Qvigstad, II 234), (Sørfj.) duogin (ebd. IV 404), (Storfj.) duogin (ebd. III 276) (Enont.) тu.oHken (Lagercrantz, LW s.v. 949), (Storfj.) duokkin (Qvigstad, LES IV 144), (Kautok.) Dùghkìin D $^{u}$ ohkien,

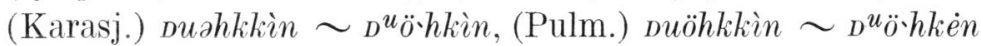
(Nielsen, Dict. 590a); I tuäha 〜 tuáh, tyehin (Wb.); R (Paatsj.)

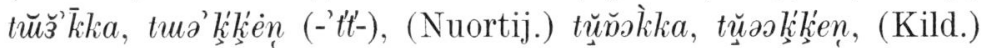

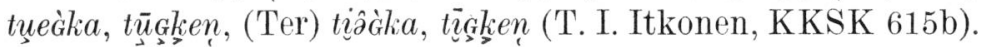

Nach dem Deklinationstyp des zweiten Gliedes wird die Form ol'gobcelen auf dem sich vom Südlappischen bis zur Südgruppe des Norwegischlappischen erstreckenden Gebiet wie eine gewöhnliche Zusammensetzung behandelt, die Form olgobcel'de von der Linie Lyngen - Kautokeino des Norwegischlappischen bis zum Kolalappischen hingegen wie eine Postpositionskonstruktion (in der Ostgruppe des Norwegischlappischen und im Inarilappischen scheint der Typ olgubcel'de den Typ olgobal'de beinahe zu überdecken).

Die Deutung des ersten Gliedes des Typs olgubcel'de als Genitiv des Substantivs wird anscheinend auch durch die Tatsache gestützt, dass im Norwegischlappischen die als Bestimmungsglied fungierende Adjektivableitung auch dann kontrahiert ist, wenn die Zusammensetzung nominativförmig ist, z.B. bâja-bcellě 'Oberseite; Oberfläche', bâja-gceččĕ 'das 
obere Ende, die obere Kante; der oberste Teil, die obere Hälfte' (Nielsen, Dict. I 110b), olgu-balle -ballě 'Aussenseite', olgu-

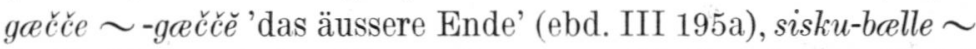

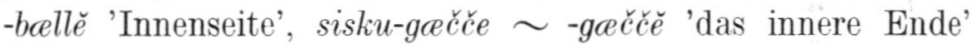
(ebd. III 413a), vuola-balle -ballě 'Unterseite' (ebd. III 802b). Dieser Genitiv lässt sich jedoch mühelos als sekundär erklären: nachdem die Kongruenzverhältnisse verdunkelt worden sind, ist er auch in diese Position eingedrungen. Das ältere Stadium findet sich bei Leem: siskadzh balle 'die innere Seite' (Nielsen, Dict. V 75a). Gleichartige und ebenso zu deutende Beispiele sind auch im Skoltlappischen zu finden: (Paats.)

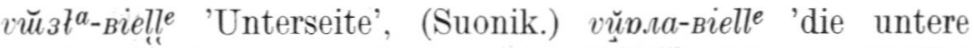
Hälfte (des Netzes, des Schleppnetzes)' (T. I. Itkonen, KKSK 786a), vgl. (Nuortij.) (E.) *ovdiš piell 'Vorderseite' (ebd. 325b).

Die inarilappischen Formen sind eindeutig kongruent und ihr erster Teil lässt sich nicht als Genitivform eines substantivisch verwendeten Adjektivs erklären. Im Lichte des Inarilappischen wird auch deutlich, dass auch die entsprechenden norwegischlappischen und skoltlappischen Formen kongruent sind. Im Inarilappischen ist die Kongruenz der $\check{5}$-Adjektive nämlich immer noch wirksam, wie Itkonen in dem erwähnten Artikel bemerkt (CSIFU I 205), z.B. paajaa ž-peeti 'Oberseite': paajaa-peln 'oberhalb (wo?)', vuoluž-peeli 'Unterseite': vuoluupeln 'unter halb (wo?)', maaddiž-keeči : (Nom.Pl.) maddiih keeijih 'die Uferseite des Netzes' (Wb.). Auch im Russischlappischen finden sich schwache Spuren dieser Kongruenz, z.B. (Nuortij.) (E.) *ovdiš piell 'Vorderseite': *out̃a passepeivast 'vom vorigen Sonntag' (T. I. Itkonen, KKSK 325b). Ebenso gibt es aus dem Süd- und Umelappischen Beispiele für die Kongruenz: S

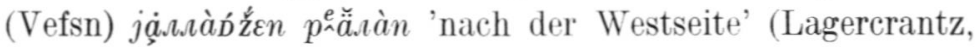
Sprachl. 43), (Hatfjelld.) nuorhtečinniä piälisne 'auf der Nordseite' (Halász, SLNy III 20); U (Malå) årjàtjòv bielèu 'zur Südseite' (Schlachter, Wb. 186).

Im Kolalappischen fehlt der Typ olgubæl'de. An Stelle der *ńD́źă Adjektivableitungen der anderen Mundarten erscheinen hier $m$-Ableitungen desselben Grundwortes, z.B. (Kild.) ${ }^{v} u \partial \bar{r} j e m-b^{i} e^{\bar{d}} t_{t}$ 'auf, von der Nordwestseite', -béàııa 'auf die Nordwestseite' (T. I. Itkonen, K KSK 793b), vùstam-веілıлa 'auf die Luvseite' (Erkki Itkonen, Sprach- 
proben, Mskr.), (Endj.) *manem biala 'hinter (wohin?)' (T. I. Itkonen, KKSK 239b), *avdtembel't 'auf, von der Vorderseite' (ebd. 367b), (Gen.) *alkem-bie $e_{i}$ t 'ausserhalb, von ausserhalb' (ebd. 318a), (Ter) (Gen.) *nirttim-pealla 'nach Süden' (ebd. 289b), *midembeäl 'auf die Leeseite' (T. I. Itkonen, KKS 319), (Endj.) *adjimbelt 'auf, vom der Vorderseite' (T. I. Itkonen, KKSK 325b), (Gen.) *savjim-pie $l$ lt 'im, vom Südwesten' (ebd. 478b). Zu diesem Typ vgl. I selgim-peeli Rückseite des Fausthandschuhs, Wollhandschuhs' < selgi 'Rücken' (Wh.), S (Åsele) vuolama-bielie 'die untere Hälfte (der Renhaut)' (Bergsland, Gramm. 204). Im Kildinlappischen gibt es auch einen Typ, in dem dem $m$ -

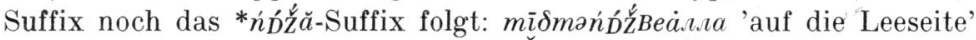

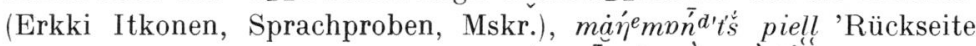

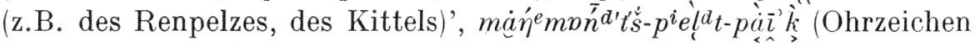
des Rens) (T. I. Itkonen, KKSK 895b). Dieser Typ enthält den in derartigen, räumliche Verhältnisse ausdrückenden Adjektiven allgemeinen Superlativcharakter (Erkki Itkonen, Stud. Ethnogr. Uppsaliensia XXI 159 ff). Es gibt Spuren der Kongruenz: (Kild.) (Tšernj.) *manma leinast 'aus dem letzten. Tuch' (ebd. 240a).

Da für die Kongruenz der *ńńžă-Ableitungen Belege vom Süden bis in den Osten vorhanden sind, dürfte die Schlussfolgerung näher liegen, dass die Kongruenz dieser Adjektivattribute auf das Urlappische zurückgeht als die, dass in den verschiedenen Dialekten voneinder unabhängige analoge Entwicklungen eingetreten sind. In diesen Partikeln liegt insofern ein einzigartiger Fall vor, als die Kongruenz durchweg eine Vollkongruenz gewesen ist. (Ein anderes gleichartiges Beispiel kann die Konnektion dâđe miel'de sein. In diesem Fall erstreckt sich die für das Ostlappische typische Partitivrektion einiger Postpositionen nach Westen, z.B. R (Kild) $t \bar{a}_{i} \delta$ mītt 'deshalb'

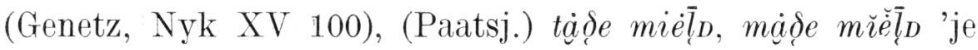
nachdem wie' (T. I. Itkonen, KKS 141); I tademield kuo 'soweit als' (A. V. Koskimies - T. Itkonen, IK 15); N dâde dâďĕ miel'dě go 'je nachdem wie, so wie; da, weil' (Nielsen, Dict. I 465a); L tatā-mit'te $\sim$ tatē-mil'te 'wieder; im selben Augenblick; darauf' (Grundström, Wb. 1091a). Vielleicht konnte sich die alte Kongruenz in diesen fest gefügten Wortverbindungen erhalten.) Im Norwegischlappischen und im Ostlappischen ist die Kongruenz dieser Adjektive auf dieselbe Art vereinfacht worden wie die Kongruenz der pronominalen Attribute, und in diesen Partikeln scheint die Richtung des 
Ausgleichs auch besonders motiviert: z.B. gibt es heute im Inarilappischen die ausgeglichene Reihe ovdiipele (Gen.), ovdiipel (Lat.), ovdiipeln (Lok.) an Stelle der den ursprünglichen Zustand vertretenden Reihe *ovdiipele, *ovdiipel, *oudă̌inpeln (der Schwund von ńńz war ja nur vor geschlossener Silbe möglich).

Aus dem Inarilappischen sind Beispiele für eine umfassendere Kongruenz des Adjektivattributs angeführt worden (Bergsland, Stud.Sept. V 34, Erkki Itkonen, CSIFU I 206). Bergsland vermutet finnischen Einfluss. Itkonen vertritt die Ansicht, dass die mit den kongruierenden Adjektivattributen verbundene Bedeutungsnuancierung nicht durch finnischen Einfluss erklärt werden kann. Nach Itkonen bekommt das Adjektiv durch die Kongruenz besonderes Gewicht, psychologischen Nachdruck.

Sowohl in Itkonens wie in Bergslands Beispielen fällt auf, dass sie auf zwei Satztypen beschränkt sind: die Beispielsätze must lii tärkki pisso 'ich habe eine genau schiessende Büchse', kal tust laa häälbih hadeh 'bei dir sind wirklich niedrige Preise', tobbeen lijiii čūutij mučcadeh visteh sunnuu viiuvast 'dort hatte ihr Schwiegersohn sehr schöne Zimmer' vertreten den einen, das Beispiel kunagas ij leem oajnàm vala nūut muččadijd viistijd lio tobbeen lijjii 'der König hatte noch nicht so schöne Zimmer wie dort waren gesehen' den anderen Typ. Im ersteren ist das leđe-Verb Prädikat, ausser dem Subjekt-Substantiv enthält dieser Typ ein sich auf das Subjekt beziehendes Adjektivglied und eine habitive Lokalkasusbestimmung. Dieselben Glieder enthält die erweiterte Prädikativkonstruktion, die ausser der Prädikativkonstruktion (Subj. + leđe + Prädikativadj.) ein die ganze Konstruktion bestimmendes habitives Glied enthält (habitive Adverbiale im Lokativ + [Sub. + leđe + Prädikativadj.]), z.B. te tust lii jyelgi tiervas 'so ist dein Fuss gesund' (Sabmelaš 126-127), nieyrih tust laa čalmeh 'deine Augen sind schlecht' (IK 131). Das Prädikativadjektiv hat also keinen festen Platz; es kann auch unmittelbar vor dem Subjekt stehen - und dann als Attribut empfunden und betont werden - : must lii tärkki pisso. In dieser Stellung 
ist es besonders hervorgehoben. Ebenso ist es auch im Finnischen: wenn das Prädikativum des Satzes hänellä on kahvi laihaa 'bei ihr ist der Kaffee dünn' vor das Subjekt gerückt wird: hänellä on laihaa kahvi, bekommt das Adjektiv mehr Nachdruck. In den Beispielen für den ersten Typ handelt es sich offensichtlich nicht um ein Attribut, sondern um ein subjektivisches Prädikativum, worauf auch die prädikative Form des Adjektivs hinweist. Im zweiten Typ wiederum handelt es sich entweder um ein objektivisches Prädikativum oder wenigstens um eine Anlehnung an ein Prädikativum. Im Inarilappischen ist die Kategorie der Prädikatsverben, die in einer ein objektivisches Prädikativum enthaltenden Konstruktion vorkommen, verhältnismässig gross; das Prädikativadjektiv ist immer von den Adverbien nūut 'so', nääyt 'so' bestimmt, z.B. mondiet tun anah akš-noouda nüut kuhe? 'warum hast du den Axtstiel so lang?' (IK 63), mondiet ostih aaimijd nääyt cibinajd? 'warum kauftest du die Nadeln so klein?' (Wb.), tot vuáša nūut kojda tom käähvi 'sie kocht den Kaffee so dünn' (Wb.). Auch in dieser Konstruktion scheint das Adjektiv keinen festen Platz zu haben, obwohl es meistens nach dem Objekt steht. Ebenso ist die Situation im Finnischen, vgl. hän keittää kahvin nïn laihaa hän keittää nïn laihaa kahvin 'sie kocht den Kaffee so dünn'; im letzteren Falle liegt auf dem Adjektiv besonders viel Nachdruck. Obwohl die inarilappischen Konstruktionen Entsprechungen im Finnischen haben, braucht man doch keinen finnischen Einfluss anzunehmen. Diese Prädikativkonstruktionen beschränken sich nicht auf das Inarilappische (sie kommen wenigstens auch im Norwegischund Lulelappischen und offenbar noch weiter vor). Im folgenden lulelappischen Beispiel handelt es sich ebenfalls um eine Prädikativkonstruktion, obwohl das unmittelbar vor dem Objekt stehende objektivische Prädikativum wie ein Attribut aussieht: uksa-tsakkeeh piejahtuvvih särroot, man kaljeev uvhsav sihtaa 'die Türpfosten setzt man auseinander, je nachdem, wie breit man die Tür haben will' (Pirak, 135).

RAIJA BaRTENS 